\title{
The Development of Engineering Vehicles Steer - by- Wire System
}

\author{
Chang Jiangxue ${ }^{1, a^{*}}$, Li Manjiang ${ }^{1, b}$, Bai Xuefeng ${ }^{2, c}$ \\ ${ }^{1}$ College of Engineering Technology, Jiangsu Institute of Commerce, Nanjing 211168, China \\ ${ }^{2}$ Jiangsu Agricultural Machinery Testing Station, Nanjing 210017,China \\ acjxsnow@126.com, ${ }^{\mathrm{b}} \mathrm{lky} 536 @ 126 . c 0 m,{ }^{\mathrm{c}}$ whatwhat521@126.com
}

\begin{abstract}
Keywords: Steer-by-wire System; Engineering Vehicles; Active safety
Abstract. Steer-by-wire is the latest technology in vehicle steering system. This paper reviews the domestic and foreign development of steer-by-wire steering system. The structure ,characteristics and working principle of the steer-by- wire steering system were analyzed in detail. At last, the solutions that making steer-by-wire steering system in engineering vehicle were proposed to improve the steering characteristics and safety of the vehicle.
\end{abstract}

\section{Introduction}

Trends in vehicle is safety, energy saving and environmental protection. The steering system, which affects vehicle's active safety, is very important. The quality of its stability have greatly effect on the performance of the vehicle. Steering system is a specialized body for changing the traveling direction of the vehicle, its function is to provide the best conditions in the various road sense, and transmits the intention of the driver to the vehicle. Its performance directly affects vehicle stability, safety and energy-saving environmental protection. Therefore, the study of steering system has not been interrupted.

The object of this paper is engineering vehicle's steering system. Due to poor working conditions, engineering vehicles often travel on rugged land, so there are special requirements for its steering system. Engineering vehicle's steering system mainly includes hydraulic steering and hydraulic power steering. The full hydraulic steering system has the advantage of flexibility, lightweight, performance stably and convenient layout, but there was often many faults during steering, like steering heavy and failure.

In recent years, the car began to use steer-by-wire technology. Due to the cancellation of the mechanical connection between the steering wheel and front wheel, steer-by-wire system, abbreviated as SBW, completely get rid of the limitations of traditional steering system. Not only the force transfer characteristics of the car can be freely designed, but also the angle transfer characteristics of the car, which bring infinite space to the design of the vehicle steering characteristics. This is a major innovation in automotive steering systems. SBW is one of the most advanced and cutting-edge technology in terms of steering. It is the development of the future steering system, and it can also applied to engineering vehicles.

\section{The development of SBW system at home and abroad}

SBW system was first used in aircraft control systems, it has been widely used on the plane. The driver control signal are converted into an electrical signal, and it is passed to the various execution units of the aircraft to control the attitude of the aircraft.

Similarly, steer-by-wire system will also improve cars' operating performance when it is applied on the car. SBW system has been proposed back in the fifties of the last century. The idea is to use an electrical signal instead of the original mechanical connection between the steering wheel and the wheel, and make electronic actuators to achieve the wheel's steering. Due to limitations of the electronic technology, it was unable to be applied to the actual vehicle.

The study of SBW system abroad has reached a certain level. Benz began to study the front wheel steering in 1990, and applied the developed steer-by-wire system on the concept car F400 Carving; 
Delphi has developed its own front and four-wheel SBW system; The concept car BMWZ22 also applied steer-by-wire system, the system made a maximum range of the steering wheel designed to $160^{\circ}$, which greatly reduced the workload of the driver during emergency steering; Italian Bertone's concept car "FILO", Citroen SUV "C-Crosser", and Daimler chrysler's concept car "R129" have also adopted SBW system; Japan's Koyo company developed SBW system too, but in order to ensure the safety of the system, it still retained the mechanical parts between the steering wheel and the wheel, the purpose is to restore to mechanical steering when steer-by-wire system failure. The research institutions abroad were also strongly involved in the study of SBW system. Such as Stanford University's hardware in the loop test and active steering control strategy, New York State University's people-vehicles closed-loop control.

However, due to the existing regulations in Europe, the cars with steer-by-wire system which has not mechanical connection are not allowed to use on public transport road, so steer-by-wire system with purely electronic control is still in the development stage and the concept stage. With the development of electronic technology, maybe a few years later, the mechanical system will be replaced by electronic signal cable, and SBW cars will become mainstream.

Domestic study of steer-by-wire have a late start. The independently developed " the 3rd Chunhui Claymore" mini electric car by Tong Ji University is representative. The car was on display at the 2005 Shanghai International Auto Parts \& Supplies Exhibition. Its biggest highlight was the adoption of steer-by-wire technology, and it was four-wheel-drive steer-by-wire tiny concept car; Beijing Institute of Technology have done a depth inquiry for steer-by-wire system's variable ratio; Jilin University conducted the study of the variable gear ratio, torque of the steering wheel and stability of the algorithm; Wuhan University did research on variable gear ratio and fuzzy control algorithm of road sense, and made a simulation about it. But these studies are still at an early stage, they have not been applied on a real car. In spite of it, Jilin University designed a steer-by-wire test, which is shown in Figure 1.

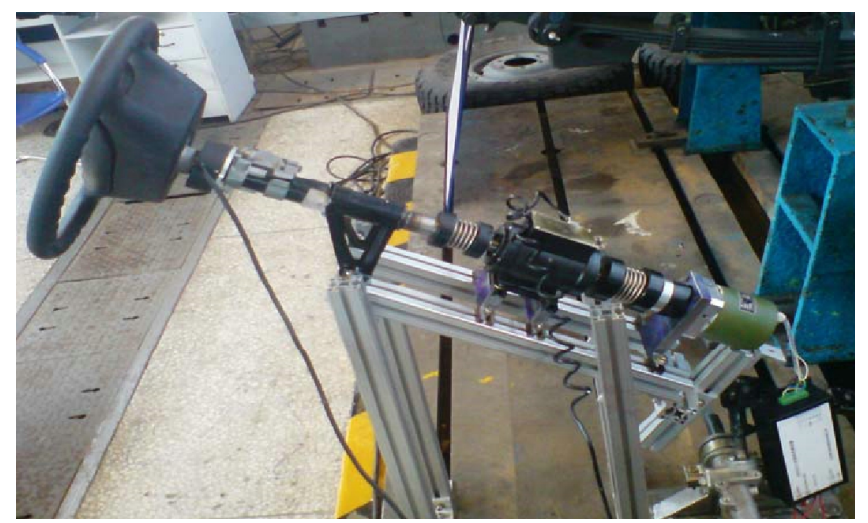

Fig.1 SBW test

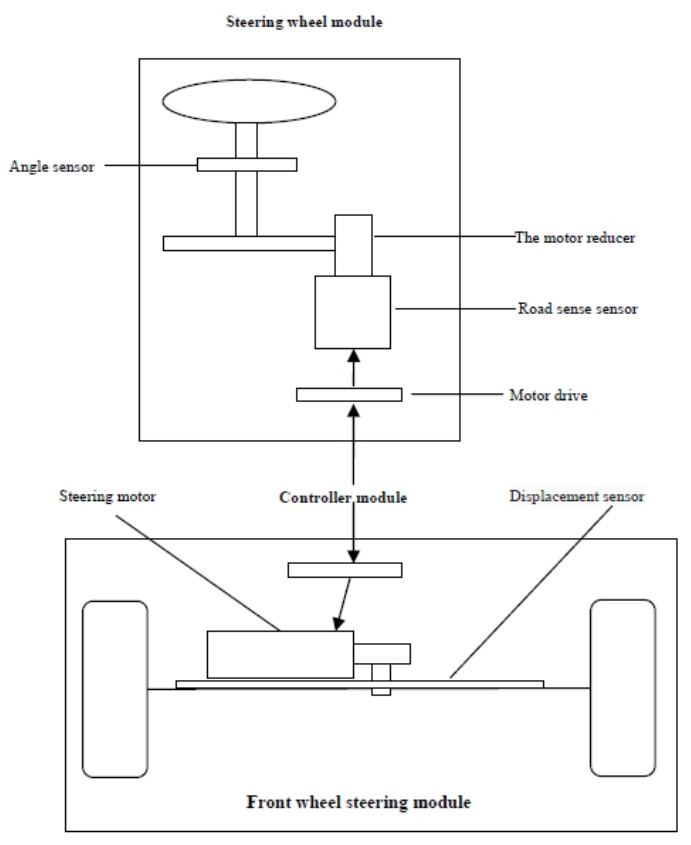

Fig.2 The structure of SBW system

\section{Structural analysis of SBW}

Steer-by-wire system changed the original steering system's transmission structure. The system operated by the driver and the wheel's execution system were completely mechanically separated, and they made up two independent subsystems. The SBW system combined this two subsystems together by its control system and sensors. Control system take the initiative to adjust the power transfer characteristics of automotive steering by analyzing and calculating the state of the vehicle, it can also 
design the angle transfer characteristics of the car, which brought infinite space to the design of the vehicle steering characteristics.

As is shown in Figure 2, SBW system consists of three modules: the steering wheel module, front wheel steering module and the controller module.

\section{The steering wheel module}

Steering wheel module includes steering wheel, steering wheel angle sensors, torque sensors, steering wheel aligning torque motor. The main function of the steering wheel module is to pass the driver's steering intent to the master controller, and then to control the operation of the steering module by the ECU; In the same time, the ECU send control signal to steering wheel aligning torque motor, which makes it produce the aligning torque to provide the driver with the road sense information.

\section{Front wheel steering module}

The front wheel steering module is composed of the front wheel angle sensor, steering implementation motor, steering motor controller, front wheel steering components and so on. The function of the steering assembly is to accept the master controller commands, and control the front wheel's turning by the steering motor controller, which can realize the driver's steering intent.

\section{The controller module}

The main function of the ECU is to collect sensor signal, and analyze the signal by the pre-set control strategy, and then sends commands to the steering wheel aligning torque motor and steering motor to control two motors' work. ECU should ensure that there must be the ideal vehicle response in a variety of conditions.

\section{SBW steering system's work characteristics}

SBW system canceled mechanical connection between the steering wheel and the front wheels, and control their moving by ECU. This can achieve the steering function that the conventional steering system can't. Steer-by-wire steering system has the following characteristics.

\section{Improving the steering features}

SBW system can set the angle transmission ratio in real-time according to vehicle's driving conditions, and at the same time compensate the front wheel angle to make sure that the vehicle's steering characteristics remain still. With the removal of the mechanical connection, SBW system can freely design the steering ratio. When the car is at low speeds, the steering ratio should be reduced, which can improve the steering sensitivity. Conversely, the steering ratio should be increased when vehicle is at high speeds, which can make the steering more stable. SBW handed over the work that should be completed by the driver to the controller, thus can reduce the driver's intensity and improve the steering system's ability to respond to steering input from the driver.

\section{Improving road sense}

Road sense refers to the turning resistance torque during the driving, which is got from the driver's feeling from the steering wheel's feedback. This torque contains the vehicle and tire's information about movement and force status, which is very important for the driver to control the vehicle motion and the environmental conditions.

In the conventional vehicles, the driver felt road sense through the steering wheel's reaction. Since there is no mechanical connection between the steering wheel and front wheel in SBW system, the driver can not feel the reaction the steering wheel, and the road sense that the driver needs is generated from control signals supplied by the steering wheel aligning torque motor. Because ECU can choose the best signal to reflect the real running state of the vehicle and the road conditions, the diver can be provided with the most useful information, and feel his favorite road sense furthermore.

\section{Improving economic efficiency and protecting the environment}

Due to cancelling the mechanical connection, SBW have reduced the weight of the vehicle, and the motor output the power only when turning. The system eliminates the belt drive and improves the transmission efficiency. Therefore, vehicle fuel economy has been improved, energy has been saved, emissions has been reduced. Seen in this light, the study of SBW has a huge and far-reaching impetus 
for the development of vehicle manufacturing industry, it represents the future of vehicle steering system.

\section{Enhancing stability and security}

Vehicle was often encounter a variety of complex conditions, such as off road, road side winds and low adhesion and so on, it can be easily unstable when the vehicle is traveling in these conditions. The SBW system can judge the state of vehicles by collecting sensor signals, and when the vehicle has unstable trend, it can control the front wheel's angle, and then control the car back to the steady state.

\section{Expanding research space and reducing development costs}

SBW system canceled the steering column, pulleys, belts and other components, so the cab has more space to arrange the passive safety components, which can reduce the harm to the driver when the accident occurred. Since the hardware part has great versatility, the system uses software control, and it can be applied to other models if you modify some parameters. And this can save a lot of time for developing new models, and reduce the costs.

\section{The outlook of SBW}

With the development of technology, people have more demand for vehicle environmental protection, energy conservation, safety and comfort. However, automotive technology has been perfect, and it is difficult to change the mechanical structure of the car to make its characteristics greatly improved, so the automotive electronics technology has become the trend of modern automotive. SBW system will eventually replace the current traditional mechanical steering system, and it has gradually been accepted by the automotive industry.

As it to the engineering vehicle, if SBW is added in fully hydraulic steering system, the electrical and fluid will combine together and form a hydraulic steer-by-wire system. This will not only have the advantage of large hydraulic power, but also under the control of the electrical components, complete and flexible steering operation., which can make the steering system's structure simple, steering flexible, accurate and reliable, and improve vehicle's handling performance. It is also a subject that engineering vehicles steering system should study for.

\section{References}

[1] Yu Leiyan, Lin Yi, Li Yufang.. Summary of automotive's steer-by-wire system. Agricultural Equipment \& Vehicle Engineering, 2006.1.

[2] Zhu Yajun. Study on structure and properties of the system of electronic steering. Xi'an: Chang'an University. (2006).

[3] Li Yi-ran, Huang Jian-ming, He Zhi-ming. A Nonlinear Control Method of Steering Angle Following Used in Steer-by-wire System. Applied Mechanics and Materials. 190-191 (2012) 890-893. [4] Ma Haibo, Yang Ying. Electrical power assisted steering for EVs and HEVs. Engineering Sciences. 9(1) (2011) 60-65.

[5] Liu Yong.Research of vehicles steer-by-wire system.. Wuhan University of Technology,2005.d tables must be in English. 